\title{
ANALYTIC SOLUTION FOR RETURN OF PREMIUM AND ROLLUP GUARANTEED MINIMUM DEATH BENEFIT OPTIONS UNDER SOME SIMPLE MORTALITY LAWS
}

\author{
BY
}

\author{
ERIC R. ULM ${ }^{1}$
}

\begin{abstract}
Much attention has been focused recently on the issue of valuing guaranteed minimum death benefits embedded in annuity contracts. These benefits resemble a sequence of put options and their value should obey a differential equation similar to the Black-Scholes equation for simple put options. This paper derives a number of analytic solutions to this equation for a number of simple mortality laws.
\end{abstract}

\section{KEYWORDS}

Guaranteed Annuity Options, Black-Scholes Equation.

\section{INTRODUCTION}

The valuation of Guaranteed Minimum Death Benefit (GMDB) riders embedded in variable annuity contracts has been a hot topic in recent years. In the main contract, a premium is paid that accumulates a sum of money on a tax deferred basis which can be converted to a payout annuity upon retirement. The initial premium can be invested in a number of different types of funds, usually including both a fixed fund earning a guaranteed rate of interest and a variable fund invested in equities.

In order to differentiate their products, variable annuity issuers have added a number of different types of riders, including GMDBs. These riders guarantee some minimum benefit at death, which is frequently the return of premiums paid and sometimes includes the accumulation of those premiums at a fixed interest rate. This situation is commonly called a "rollup" GMDB. Because

1 Eric Ulm, FSA, is an Assistant Professor in the Department of Risk Management and Insurance, J. Mack Robinson College of Business, Georgia State University, Atlanta, GA. Phone: 404-413-7485. E-mail: inseuu@langate.gsu.edu. Some work performed while at the University of Central Florida, Orlando, FL. 
the underlying assets are invested in stock funds, GMDB riders resemble a sequence of European put options, with the put value on a given date being multiplied by the probability the annuitant dies that date and has not yet lapsed his policy. The integral that determines the value of the option can be written as follows (see, for example, Hardy (2003)),

$$
\begin{aligned}
& f_{a}(S, t)= \\
& \int_{0}^{\infty}\left\{X e^{p t} e^{p w} e^{-r w} N\left[-d_{2}(S, w)\right]-S e^{-q w} N\left[-d_{1}(S, w)\right]\right\} \mu(t+w)_{w} p_{x+t}^{(\tau)} d w
\end{aligned}
$$

where $X$ is the strike at issue, $p$ is the roll-up rate, $r$ is the risk-free rate, $S$ is the stock level today, $q$ is the level of asset fees, $x$ is the age at issue and $t$ is the years since issue. Also:

$$
\begin{aligned}
& d_{1}(S, w)=\frac{\ln \left(\frac{S}{X e^{p t}}\right)+\left(r-q-p+\frac{\sigma^{2}}{2}\right) w}{\sigma \sqrt{w}} ; \\
& d_{2}(S, w)=\frac{\ln \left(\frac{S}{X e^{p t}}\right)+\left(r-q-p-\frac{\sigma^{2}}{2}\right) w}{\sigma \sqrt{w}}
\end{aligned}
$$

A number of papers address issues specific to GMDB pricing, including Milevsky and Posner (2001) and Milevsky and Salisbury (2001), which derives a differential equation that must be satisfied by the GMDB value. A number of solutions have been found assuming constant mortality and lapse for return of premium GMDBs, both in that paper and by Ulm (2006). This paper extends those results to roll-up GMDBs. In addition, solutions are found for term insurance and endowment insurance under constant force of mortality. Finally, a solution is found if mortality follows DeMoivre's Law.

\section{The Differential Equation To Be Solved}

Assume the existence of a deferred annuity with a variable account. The annuity has a GMDB rider that guarantees a return of premium with $p \%$ interest compounded continuously upon the death of the annuitant. This could be modeled as the sum of a continuous sequence of European put options (see, for example, Hardy (2003)). The weight at a given option duration would be equal to the instantaneous probability of death at that moment. The value of the strike at that moment would be $X e^{p t}$, where $X$ is the value of the initial premium paid. The equation that must be satisfied by the value of the GMDB, $f_{a}(S, t)$, is: 


$$
\begin{aligned}
\frac{\partial f_{a}}{\partial t}+(r-q) & S \frac{\partial f_{a}}{\partial S}+\frac{1}{2} \sigma^{2} S^{2} \frac{\partial^{2} f_{a}}{\partial S^{2}} \\
= & {\left[r+\mu_{x}(t)+\lambda(S, t)\right] f_{a}-\left[\mu_{x}(t)\right] \operatorname{Max}\left(X e^{p t}-S, 0\right) }
\end{aligned}
$$

which is derived in Milevsky and Salisbury (2001), or Ulm (2006) assuming that the stock fund follows a geometric Brownian motion with standard deviation $\sigma . f_{a}(S, t)$ is the value of the option if the individual is alive and has not lapsed his policy. $q$ represents the asset fees that are taken out of the account. $\mu_{x}(t)$ is the force of mortality for a person age $(x)$ and $\lambda(S, t)$ is the lapse rate, which could in theory depend on both the stock level and time. In the usual Black-Scholes differential equation, the hedge must grow at the risk free rate. In deriving Equation (3), it is the expected value of the option that must be hedged, which consists of both the live value of the option and the expected value of any benefits paid out on death or surrender. This accounts for the larger factor multiplying the value of $f_{a}(S, t)$ on the right hand side of Equation (3), as well as the additional subtracted term. Equation (3) can be derived by constructing a riskless portfolio of GMDB riders and underying stock which must earn the risk-free rate, as in the derivation of the standard BlackScholes equation. Alternatively, the integral in equation (1) can be shown to satisfy equation (3).

\subsection{General Solution to Equation (3).}

Assuming a constant lapse rate $\lambda(S, t)=\lambda$, but without making any assumptions on $\mu_{x}(t)$, it can be shown that

$$
f_{a}(S, t)=\left\lfloor X e^{p t} A(t)-S B(t)\right\rfloor H\left(X e^{p t}-S\right)+X e^{a y} f(\tau) w(y, \tau)
$$

with the following definitions: $y=\ln \left(\frac{S}{X}\right)$ and $\tau=\frac{\sigma^{2}(T-t)}{2}$ are dimensionless variables. $A(t)$ and $B(t)$ are $\bar{A}_{x+t}$ (single premium for a fully continuous whole life policy on an $x+t$ year old) at force of interest $r+\lambda-p$ and $q+\lambda$ respectively. $H(x)$ is the Heaviside step function.

$$
\begin{gathered}
\alpha=\frac{1}{2}-\frac{(r-q)}{\sigma^{2}} \\
f(\tau)=f\left(\tau_{0}\right) e^{-\kappa\left(\tau-\tau_{0}\right)} e^{-\frac{2}{\sigma^{2}} \int_{\tau_{0}}^{\tau} \mu(s) d s}, \tau_{0} \text { and } f\left(\tau_{0}\right) \text { arbitrary }
\end{gathered}
$$

And 


$$
\begin{aligned}
w(y, \tau) & =\frac{\alpha+\beta}{\sqrt{4 \pi}} e^{(1-\alpha) p T} \int_{0}^{\tau} e^{(\alpha-1) \beta s} \frac{A(s)}{f(s)} \frac{e^{-(y-[p T-\beta s])^{2} / 4(\tau-s)}}{\sqrt{\tau-s}} d s \\
& -\frac{\alpha+\beta-1}{\sqrt{4 \pi}} e^{(1-\alpha) p T} \int_{0}^{\tau} e^{(\alpha-1) \beta s} \frac{B(s)}{f(s)} \frac{e^{-(y-[p T-\beta s])^{2} / 4(\tau-s)}}{\sqrt{\tau-s}} d s \\
& +\frac{e^{(1-\alpha) p T}}{4 \sqrt{\pi}} \int_{0}^{\tau} e^{(\alpha-1) \beta s} \frac{A(s)}{f(s)}(y-[p T-\beta s]) \frac{e^{-(y-[p T-\beta s])^{2} / 4(\tau-s)}}{(\tau-s)^{3 / 2}} d s \\
& -\frac{e^{(1-\alpha) p T}}{4 \sqrt{\pi}} \int_{0}^{\tau} e^{(\alpha-1) \beta s} \frac{B(s)}{f(s)}(y-[p T-\beta s]) \frac{e^{-(y-[p T-\beta s])^{2} / 4(\tau-s)}}{(\tau-s)^{3 / 2}} d s
\end{aligned}
$$

where $T$ is either an endowment age, or the age at which the force of mortality becomes infinite, and $\beta=\frac{2 p}{\sigma^{2}}$. The details of the derivation are in Appendix A.

\section{Solution to Equation (3) Under Specific Mortality Laws}

\subsection{The Value of a Roll-Up GMDB under De Moivre's Law Mortality}

The general procedure for determining the value of the rollup is to find $A(t)$, $B(t)$ and $f(t)$, substitute them into Equation (7), and then compute the integrals. For De Moivre's Law, $\mu(t)=\frac{1}{T-t}$ so $\mu(t)=\frac{\sigma^{2}}{2 \tau}$.

also:

$$
\begin{aligned}
& A(\tau)=\frac{\sigma^{2}}{2(r+\lambda-p) \tau}\left[1-e^{-\frac{2(r+\lambda-p)}{\sigma^{2}} \tau}\right] \\
& B(\tau)=\frac{\sigma^{2}}{2(q+\lambda) \tau}\left[1-e^{-\frac{2(q+\lambda)}{\sigma^{2}} \tau}\right]
\end{aligned}
$$

also, from Equation (6) with the arbitrary constant $\tau_{0}$ chosen to satisfy $\tau_{0} e^{\kappa \tau_{0}}=1$, and $f\left(\tau_{0}\right)=1$ :

$$
f(\tau)=\left(\frac{e^{-\kappa \tau}}{\tau}\right)
$$

Substituting Equations (8), (9) and (10) into Equation (7) yields the following set of integrals for $w(y, \tau)$ : 


$$
\begin{aligned}
& w(y, \tau)=(\alpha+\beta) e^{(1-\alpha) p T} \frac{\sigma^{2}}{2(r+\lambda-p)}\left[\int_{0}^{\tau} \frac{e^{[\kappa+(\alpha-1) \beta] s} e^{-(y-[p T-\beta s])^{2} / 4(\tau-s)}}{\sqrt{4 \pi(\tau-s)}} d s\right. \\
& \left.-\int_{0}^{\tau} \frac{e^{\left[\alpha^{2}+\alpha \beta\right] s} e^{-(y-[p T-\beta s])^{2} / 4(\tau-s)}}{\sqrt{4 \pi(\tau-s)}} d s\right] \\
& -(\alpha+\beta-1) e^{(1-\alpha) p T} \frac{\sigma^{2}}{2(q+\lambda)}\left[\int_{0}^{\tau} \frac{e^{[\kappa+(\alpha-1) \beta] s} e^{-(y-[p T-\beta s])^{2} / 4(\tau-s)}}{\sqrt{4 \pi(\tau-s)}} d s\right. \\
& \left.-\int_{0}^{\tau} \frac{e^{\left[(\alpha-1)^{2}+(\alpha-1) \beta\right] s} e^{-(y-[p T-\beta s])^{2} / 4(\tau-s)}}{\sqrt{4 \pi(\tau-s)}} d s\right] \\
& +e^{(1-\alpha) p T} \frac{\sigma^{2}}{2(r+\lambda-p)}\left[\int_{0}^{\tau} \frac{e^{[\kappa+(\alpha-1) \beta] s} e^{-(y-[p T-\beta s])^{2} / 4(\tau-s)}}{4 \sqrt{\pi}(\tau-s)^{3 / 2}}(y-[p T-\beta s]) d s\right. \\
& \left.-\int_{0}^{\tau} \frac{e^{\left[\alpha^{2}+\alpha \beta\right] s} e^{-(y-[p T-\beta s])^{2} / 4(\tau-s)}}{4 \sqrt{\pi}(\tau-s)^{3 / 2}}(y-[p T-\beta s]) d s\right] \\
& -e^{(1-\alpha) p T} \frac{\sigma^{2}}{2(q+\lambda)}\left[\int_{0}^{\tau} \frac{e^{[\kappa+(\alpha-1) \beta] s} e^{-(y-[p T-\beta s])^{2} / 4(\tau-s)}}{4 \sqrt{\pi}(\tau-s)^{3 / 2}}(y-[p T-\beta s]) d s\right. \\
& \left.-\int_{0}^{\tau} \frac{e^{\left[(\alpha-1)^{2}+(\alpha-1) \beta\right] s} e^{-(y-[p T-\beta s])^{2} / 4(\tau-s)}}{4 \sqrt{\pi}(\tau-s)^{3 / 2}}(y-[p T-\beta s]) d s\right]
\end{aligned}
$$

We will use the following identities:

$$
\begin{aligned}
& \int_{0}^{\tau} \frac{e^{a s} e^{-(y-[p T-\beta s])^{2} / 4(\tau-s)}}{\sqrt{4 \pi(\tau-s)}} d s \\
& =e^{\frac{\beta}{2} y} e^{\frac{-\beta p}{2} T} \frac{e^{\left(a+\frac{\beta^{2}}{2}\right) \tau}}{2 \sqrt{a+\frac{\beta^{2}}{4}}}\left\{e^{-\sqrt{a+\frac{\beta^{2}}{4}}|y-p T+\beta \tau|} N\left[-\left(\frac{|y-p T+\beta \tau|}{\sqrt{2 \tau}}-\sqrt{2\left(a+\frac{\beta^{2}}{4}\right) \tau}\right)\right]\right. \\
& \left.\left.-e^{-\sqrt{a+\frac{\beta^{2}}{4}}|y-p T+\beta \tau|} N\left[-\left(\frac{|y-p T+\beta \tau|}{\sqrt{2 \tau}}+\sqrt{2\left(a+\frac{\beta^{2}}{4}\right)}\right)\right]\right]\right\}
\end{aligned}
$$

and 


$$
\begin{aligned}
& \int_{0}^{\tau} \frac{e^{a s} e^{-(y-[p T-\beta s])^{2} / 4(\tau-s)}}{4 \sqrt{\pi}(\tau-s)^{3 / 2}}(y-[p T-\beta s]) d s \\
& =e^{\frac{\beta}{2} y} e^{\frac{-\beta p}{2} T} \frac{e^{\left(a+\frac{\beta^{2}}{2}\right) \tau}(y-p T+\beta \tau)}{2|y-p T+\beta \tau|}\left\{e^{-\sqrt{a+\frac{\beta^{2}}{4}}|y-p T+\beta \tau|} N\left[-\left(\frac{|y-p T+\beta \tau|}{\sqrt{2 \tau}}-\sqrt{2\left(a+\frac{\beta^{2}}{4}\right) \tau}\right)\right]\right. \\
& \left.+e^{\sqrt{a+\frac{\beta^{2}}{4}|y-p T+\beta \tau|}} N\left[-\left(\frac{|y-p T+\beta \tau|}{\sqrt{2 \tau}}+\sqrt{2\left(a+\frac{\beta^{2}}{4}\right) \tau}\right)\right]\right\} \\
& -\frac{\beta}{2} e^{\frac{\beta}{2} y} e^{\frac{-\beta p}{2} T} \frac{e^{\left(a+\frac{\beta^{2}}{2}\right) \tau}}{2 \sqrt{a+\frac{\beta^{2}}{4}}}\left\{e^{-\sqrt{a+\frac{\beta^{2}}{4}|y-p T+\beta \tau|} N\left[-\left(\frac{|y-p T+\beta \tau|}{\sqrt{2 \tau}}-\sqrt{2\left(a+\frac{\beta^{2}}{4}\right)}\right)\right]}\right. \\
& \left.-e^{\sqrt{a+\frac{\beta^{2}}{4}}|y-p T+\beta \tau|} N\left[-\left(\frac{|y-p T+\beta \tau|}{\sqrt{2 \tau}}+\sqrt{2\left(a+\frac{\beta^{2}}{4}\right)}\right)\right] \tau\right)
\end{aligned}
$$

This leads, after much algebra, to the following solution for $f_{a}(S, t)$ :

REGION I. $S>X e^{p t}$

$$
\begin{aligned}
f_{a}(S, t) & =\frac{C_{1}}{(T-t)} X e^{p t}\left(\frac{S}{X e^{p t}}\right)^{m_{1}} N\left(-\xi_{1}\right)+\frac{C_{2}}{(T-t)} X e^{p t}\left(\frac{S}{X e^{p t}}\right)^{m_{2}} N\left(-\xi_{2}\right) \\
& +\frac{S e^{-(q+\lambda)(T-t)}}{(q+\lambda)(T-t)} N\left(-d_{1}\right)-\frac{X e^{p t} e^{-(r+\lambda-p)(T-t)}}{(r+\lambda-p)(T-t)} N\left(-d_{2}\right)
\end{aligned}
$$

and

REGION II. $S<X e^{p t}$

$$
\begin{aligned}
f_{a}(S, t) & =\frac{X e^{p t}\left(1-e^{-(r+\lambda-p)(T-t)}\right)}{(r+\lambda-p)(T-t)}-\frac{S\left(1-e^{-(q+\lambda)(T-t)}\right)}{(q+\lambda)(T-t)} \\
& -\frac{C_{1}}{(T-t)} X e^{p t}\left(\frac{S}{X e^{p t}}\right)^{m_{1}} N\left(\xi_{1}\right)-\frac{C_{2}}{(T-t)} X e^{p t}\left(\frac{S}{X e^{p t}}\right)^{m_{2}} N\left(\xi_{2}\right) \\
& +\frac{X e^{p t} e^{-(r+\lambda-p)(T-t)}}{(r+\lambda-p)(T-t)} N\left(d_{2}\right)-\frac{S e^{-(q+\lambda)(T-t)}}{(q+\lambda)(T-t)} N\left(d_{1}\right)
\end{aligned}
$$

with definitions: 
$d_{1}=\frac{\ln \left(\frac{S}{X e^{p t}}+\left(r-q-p+\frac{\sigma^{2}}{2}\right)(T-t)\right)}{\sigma \sqrt{(T-t)}} ;$

$d_{2}=\frac{\ln \left(\frac{S}{X e^{p t}}+\left(r-q-p-\frac{\sigma^{2}}{2}\right)(T-t)\right)}{\sigma \sqrt{(T-t)}} ;$

$\xi_{1}=\frac{\ln \left(\frac{S}{X e^{p t}}\right)+\sigma^{2} \sqrt{K}(T-t)}{\sigma \sqrt{(T-t)}} ; \xi_{2}=\frac{\ln \left(\frac{S}{X e^{p t}}\right)-\sigma^{2} \sqrt{K}(T-t)}{\sigma \sqrt{(T-t)}} ;$

$m_{1}=A+\sqrt{K} ; m_{2}=A-\sqrt{K} ; A=\frac{1}{2}-\frac{(r-q-p)}{\sigma^{2}} ; K=A^{2}+\frac{2(r+\lambda-p)}{\sigma^{2}}$;

$C_{1}=\frac{1}{2(q+\lambda)}\left[\frac{A-1}{\sqrt{K}}-1\right]-\frac{1}{2(r+\lambda-p)}\left[\frac{A}{\sqrt{K}}-1\right] ;$

$C_{2}=\frac{1}{2(r+\lambda-p)}\left[1+\frac{A}{\sqrt{K}}\right]-\frac{1}{2(q+\lambda)}\left[1+\frac{A-1}{\sqrt{K}}\right]$;

The existence of distinct solution in the two regions is due to the absolute values in equation (13) combined with careful evaluation of expressions of the form $\sqrt{A^{2}}$ in the later stages of the derivation.

\subsection{The Value of a Roll-Up GMDB under Constant Force of Mortality with Endowment}

We now turn our attention to solution of Equation (3) for constant mortality. The solution for $p=\lambda=0$ was found in Milevksy and Salisbury (2001) and the solution for $p=0 ; \lambda \neq 0$ is in Ulm (2006). We now find the answer for $p \neq 0$. We will begin by finding the value assuming an endowment age corresponding to an endowment time $T$. At this age, a surviving individual receives his full GMDB. We will finally allow $T$ to go to infinity.

We will follow a procedure analogous to that in section 3.1. We must find $A(t)$, $B(t)$ and $f(t)$, substitute them into Equation (7), and then compute the integrals. In this case, $\mu(t)=\mu$ and $\mu(\tau)=\mu$. Including the effect of the endowment,

$$
A(\tau)=\frac{\mu}{\mu+r+\lambda-p}+\left(\frac{r+\lambda-p}{\mu+r+\lambda-p}\right) e^{-\frac{2(\mu+r+\lambda-p)}{\sigma^{2}} \tau}
$$

and 


$$
B(\tau)=\frac{\mu}{\mu+q+\lambda}+\left(\frac{q+\lambda}{\mu+q+\lambda}\right) e^{-\frac{2(\mu+q+\lambda)}{\sigma^{2}} \tau}
$$

also, from Equation (6) with $f\left(\tau_{0}\right)=1$

$$
f(\tau)=e^{-\kappa\left(\tau-\tau_{0}\right)} e^{-\frac{2}{\sigma^{2}} \int_{\tau 0}^{\tau} \mu d s}=e^{-(\kappa+\gamma) \tau}
$$

where

$$
\gamma=\frac{2 \mu}{\sigma^{2}}
$$

Substituting Equations (16), (17) and (18) into Equation (7) yields the following set of integrals for $w(y, t)$ :

$$
\begin{aligned}
& w(y, \tau)=(\alpha+\beta) e^{(1-\alpha) p T}\left[\frac{\mu}{\mu+r+\lambda-p} \int_{0}^{\tau} \frac{e^{[\kappa+\gamma+(\alpha-1) \beta] s} e^{-(y-[p T-\beta s])^{2} / 4(\tau-s)}}{\sqrt{4 \pi(\tau-s)}} d s\right. \\
&\left.+\frac{r+\lambda-p}{\mu+r+\lambda-p} \int_{0}^{\tau} \frac{e^{\left[\alpha^{2}+\alpha \beta\right] s} e^{-(y-[p T-\beta s])^{2} / 4(\tau-s)}}{\sqrt{4 \pi(\tau-s)}} d s\right] \\
&-(\alpha+\beta-1) e^{(1-\alpha) p T}\left[\frac{\mu}{\mu+q+\lambda} \int_{0}^{\tau} \frac{e^{[\kappa+\gamma+(\alpha-1) \beta] s} e^{-(y-[p T-\beta s])^{2} / 4(\tau-s)}}{\sqrt{4 \pi(\tau-s)}} d s\right. \\
&\left.+\frac{q+\lambda}{\mu+q+\lambda} \int_{0}^{\tau} \frac{e^{\left[(\alpha-1)^{2}+(\alpha-1) \beta\right] s} e^{-(y-[p T-\beta s])^{2} / 4(\tau-s)}}{\sqrt{4 \pi(\tau-s)}} d s\right] \\
&+e^{(1-\alpha) p T}\left[\frac{\mu}{\mu+r+\lambda-p} \int_{0}^{\tau} \frac{e^{[\kappa+\gamma+(\alpha-1) \beta] s} e^{-(y-[p T-\beta s])^{2} / 4(\tau-s)}}{4 \sqrt{\pi}(\tau-s)^{3 / 2}}(y-[p T-\beta s]) d s\right. \\
&\left.+\frac{r+\lambda-p}{\mu+r+\lambda-p} \int_{0}^{\tau} \frac{e^{\left[\alpha^{2}+\alpha \beta\right] s} e^{-(y-[p T-\beta s])^{2} / 4(\tau-s)}}{4 \sqrt{\pi}(\tau-s)^{3 / 2}}(y-[p T-\beta s]) d s\right] \\
&-e^{(1-\alpha) p T}\left[\frac{\mu}{\mu+q+\lambda} \int_{0}^{\tau} \frac{e^{[\kappa+\gamma+(\alpha-1) \beta] s} e^{-(y-[p T-\beta s])^{2} / 4(\tau-s)}}{4 \sqrt{\pi}(\tau-s)^{3 / 2}}(y-[p T-\beta s]) d s\right. \\
&\left.+\frac{q+\lambda}{\mu+q+\lambda} \int_{0}^{\tau} \frac{e^{\left[(\alpha-1)^{2}+(\alpha-1) \beta\right] s} e^{-(y-[p T-\beta s])^{2} / 4(\tau-s)}}{4 \sqrt{\pi}(\tau-s)^{3 / 2}}(y-[p T-\beta s]) d s\right]
\end{aligned}
$$

Applying Equations (12) and (13) and working through the algebra gives the following results for $f_{a}(S, t)$. 
REGION I. $S>X e^{p t}$

$$
\begin{aligned}
f_{a}(S, t) & =C_{1} X e^{p t}\left(\frac{S}{X e^{p t}}\right)^{m_{1}} N\left(-\xi_{1}\right)+C_{2} X e^{p t}\left(\frac{S}{X e^{p t}}\right)^{m_{2}} N\left(-\xi_{2}\right) \\
& +\frac{r+\lambda-p}{(\mu+r+\lambda-p)} X e^{p t} e^{-(\mu+r+\lambda-p)(T-t)} N\left(-d_{2}\right) \\
& -\frac{q+\lambda}{(\mu+q+\lambda)} S e^{-(\mu+q+\lambda)(T-t)} N\left(-d_{1}\right)
\end{aligned}
$$

and

REGION II. $S<X e^{p t}$

$$
\begin{aligned}
f_{a}(S, t) & =\frac{\mu}{(\mu+r+\lambda-p)} X e^{p t}-\frac{\mu}{(\mu+q+\lambda)} S \\
& -C_{1} X e^{p t}\left(\frac{S}{X e^{p t}}\right)^{m_{1}} N\left(\xi_{1}\right)-C_{2} X e^{p t}\left(\frac{S}{X e^{p t}}\right)^{m_{2}} N\left(\xi_{2}\right) \\
& +\frac{r+\lambda-p}{(\mu+r+\lambda-p)} X e^{p t} e^{-(\mu+r+\lambda-p)(T-t)} N\left(-d_{2}\right) \\
& -\frac{q+\lambda}{(\mu+q+\lambda)} S e^{-(\mu+q+\lambda)(T-t)} N\left(-d_{1}\right)
\end{aligned}
$$

The parameters are defined as in Equation (15), with the exceptions:

$$
\begin{aligned}
& K=A^{2}+\frac{2(\mu+r+\lambda-p)}{\sigma^{2}} \\
& C_{1}=\frac{\mu}{2(\mu+q+\lambda)}\left[\frac{A-1}{\sqrt{K}}-1\right]-\frac{\mu}{2(\mu+r+\lambda-p)}\left[\frac{A}{\sqrt{K}}-1\right] \\
& C_{2}=\frac{\mu}{2(\mu+r+\lambda-p)}\left[1+\frac{A}{\sqrt{K}}\right]-\frac{\mu}{2(\mu+q+\lambda)}\left[1+\frac{A-1}{\sqrt{K}}\right]
\end{aligned}
$$

The value of the endowment alone is given by the value of a put option at time $T$ multiplied by the probability that the person has not lapsed and is alive to receive the endowment. This value is therefore:

$$
X e^{p t} e^{-(\mu+r+\lambda-p)(T-t)} N\left(-d_{2}\right)-S e^{-(\mu+q+\lambda)(T-t)} N\left(-d_{1}\right)
$$

in both ranges. The term insurance alone is the difference between Equations (21) and (23). 
The value of a rollup GMDB under constant force of mortality at all ages can be found from Equation (21) by allowing the endowment age $T \rightarrow \infty$. This gives the following equations:

$$
\begin{aligned}
& S>X e^{p t} \quad f_{a}(S, t)=C_{2} X e^{p t}\left(\frac{S}{X e^{p t}}\right)^{m_{2}} \\
& S<X e^{p t} \quad f_{a}(S, t)=\frac{\mu}{(\mu+r+\lambda-p)} X e^{p t}-\frac{\mu}{(\mu+q+\lambda)} S-C_{1} X e^{p t}\left(\frac{S}{X e^{p t}}\right)^{m_{1}}
\end{aligned}
$$

These equations collapse to those shown in Milevsky and Salisbury (2001) for constant force of mortality and $\lambda=p=0$.

\section{NUMERICAL COMPARISONS}

We will compare the values that we obtain from these derived formulas with numeric integration of Equation (1). Two features of Equation (1) are worth mentioning. First, the interest rate $r$ only appears in the combination $r-p$. This implies that Equations (14), (21) and (24) should not involve $r$ except in the combination $r-p$ and this turns out to be true. Second, the strike $X$ only appears in the combination $X e^{p t}$, i.e. the strike at time $t$. This implies that only that combination should appear in Equations (14), (21) and (24), which is also true.

We will now compare the results of Equations (14), (21) and (24) with the results one would get from Equation (1). These results should be identical, and we include them here as a check that the analytic solution is correct.

For De Moivre's Law, Equation (1) becomes:

$$
f_{a}(S, t)=\int_{0}^{T-t}\left\{X e^{p t} e^{p w} e^{-r w} N\left[-d_{2}(S, w)\right]-S e^{-q w} N\left[-d_{1}(S, w)\right]\right\} \frac{e^{-\lambda w}}{T-t} d w
$$

Equation (25) must be evaluated using numeric techniques (although the procedure described in the preceding sections could be viewed as a very complicated way of finding an analytic solution to the integral). Table 1 gives some values of Equation (14) and Equation (25) for various values of $X, r, p, q, \sigma$, $\lambda, S$ and $T-t$.

For constant force with endowment, Equation (1) becomes:

$$
\begin{aligned}
f_{a}(S, t) & =\int_{0}^{T-t}\left\{X e^{p t} e^{p w} e^{-r w} N\left[-d_{2}(S, w)\right]-S e^{-q w} N\left[-d_{1}(S, w)\right]\right\} \mu e^{-(\mu+\lambda) w} d w \\
& +\left\{X e^{p t} e^{p(T-t)} e^{-r(T-t)} N\left[-d_{2}(S, T-t)\right]\right. \\
& \left.-S e^{-q(T-t)} N\left[-d_{1}(S, T-t)\right]\right\} e^{-(\mu+\lambda)(T-t)}
\end{aligned}
$$


TABLE 1

Comparison of Closed Form and Integration for De Moivre's LaW Mortality

\begin{tabular}{cccccccccc}
\hline \hline Strike & $\boldsymbol{r}$ & $\boldsymbol{p}$ & $\boldsymbol{q}$ & $\boldsymbol{\sigma}$ & $\boldsymbol{\lambda}$ & $\boldsymbol{S}$ & $\boldsymbol{T}-\boldsymbol{t}$ & Closed Form & Integration \\
\hline 1 & $8 \%$ & $3 \%$ & $1 \%$ & $20 \%$ & $2 \%$ & 0.5 & 20 & 0.21424733 & 0.21424730 \\
1 & $8 \%$ & $3 \%$ & $1 \%$ & $20 \%$ & $2 \%$ & 1 & 20 & 0.05555276 & 0.05555275 \\
1 & $8 \%$ & $3 \%$ & $1 \%$ & $20 \%$ & $2 \%$ & 2 & 20 & 0.00671562 & 0.00671565 \\
1 & $5 \%$ & $3 \%$ & $1 \%$ & $20 \%$ & $2 \%$ & 1 & 20 & 0.12664668 & 0.12664669 \\
1 & $8 \%$ & $1 \%$ & $1 \%$ & $20 \%$ & $2 \%$ & 1 & 20 & 0.03202345 & 0.03202346 \\
1 & $8 \%$ & $3 \%$ & $4 \%$ & $20 \%$ & $2 \%$ & 1 & 20 & 0.09328518 & 0.09328518 \\
1 & $8 \%$ & $3 \%$ & $1 \%$ & $40 \%$ & $2 \%$ & 1 & 20 & 0.16380654 & 0.16380658 \\
1 & $8 \%$ & $3 \%$ & $1 \%$ & $20 \%$ & $10 \%$ & 1 & 20 & 0.02982873 & 0.02982875 \\
1 & $8 \%$ & $3 \%$ & $1 \%$ & $20 \%$ & $2 \%$ & 1 & 40 & 0.03611639 & 0.03611640 \\
\hline \hline
\end{tabular}

TABLE 2.

Comparison of Closed Form and Integration for Constant Force of Mortality WITH ENDOWMENT

\begin{tabular}{ccccccccccc}
\hline \hline Strike & $\boldsymbol{\mu}$ & $\boldsymbol{r}$ & $\boldsymbol{p}$ & $\boldsymbol{q}$ & $\boldsymbol{\sigma}$ & $\boldsymbol{\lambda}$ & $\boldsymbol{S}$ & $\boldsymbol{T}-\boldsymbol{t}$ & Closed Form & Integration \\
\hline 1 & $2 \%$ & $8 \%$ & $3 \%$ & $1 \%$ & $20 \%$ & $2 \%$ & 0.5 & 20 & 0.12626180 & 0.12626182 \\
1 & $2 \%$ & $8 \%$ & $3 \%$ & $1 \%$ & $20 \%$ & $2 \%$ & 1 & 20 & 0.03977509 & 0.03977511 \\
1 & $2 \%$ & $8 \%$ & $3 \%$ & $1 \%$ & $20 \%$ & $2 \%$ & 2 & 20 & 0.00779967 & 0.00779952 \\
1 & $6 \%$ & $8 \%$ & $3 \%$ & $1 \%$ & $20 \%$ & $2 \%$ & 1 & 20 & 0.05063465 & 0.05063466 \\
1 & $2 \%$ & $5 \%$ & $3 \%$ & $1 \%$ & $20 \%$ & $2 \%$ & 1 & 20 & 0.12604128 & 0.12604124 \\
1 & $2 \%$ & $8 \%$ & $1 \%$ & $1 \%$ & $20 \%$ & $2 \%$ & 1 & 20 & 0.01810033 & 0.01810031 \\
1 & $2 \%$ & $8 \%$ & $3 \%$ & $4 \%$ & $20 \%$ & $2 \%$ & 1 & 20 & 0.07744060 & 0.07744057 \\
1 & $2 \%$ & $8 \%$ & $3 \%$ & $1 \%$ & $40 \%$ & $2 \%$ & 1 & 20 & 0.13253453 & 0.13253460 \\
1 & $2 \%$ & $8 \%$ & $3 \%$ & $1 \%$ & $20 \%$ & $10 \%$ & 1 & 20 & 0.01472027 & 0.01472029 \\
1 & $2 \%$ & $8 \%$ & $3 \%$ & $1 \%$ & $20 \%$ & $2 \%$ & 1 & 40 & 0.02587016 & 0.02587016 \\
\hline \hline
\end{tabular}

Again, Equation (26) is evaluated using numeric integration. Table 2 gives some values of Equation (21) and Equation (26) for various values of $X, \mu, r, p, q$, $\sigma, \lambda, S$ and $T-t$.

Finally, for constant force, Equation (1) becomes:

$$
f_{a}(S, t)=\int_{0}^{\infty}\left\{X e^{p t} e^{p w} e^{-r w} N\left[-d_{2}(S, w)\right]-S e^{-q w} N\left[-d_{1}(S, w)\right]\right\} \mu e^{-(\mu+\lambda) w} d w
$$

Table 3 gives some values of Equation (24) and Equation (27), also evaluated by numeric integration, for various values of $X, \mu, r, p, q, \sigma, \lambda$ and $S$. In all three tables, the differences are mostly due to the discreteness of the numerical integration, and the closed form answers are actually the more accurate values. 
TABLE 3.

Comparison of Closed Form and Integration for Constant Force of Mortality at all ages.

\begin{tabular}{cccccccccc}
\hline \hline Strike & $\boldsymbol{\mu}$ & $\boldsymbol{r}$ & $\boldsymbol{p}$ & $\boldsymbol{q}$ & $\boldsymbol{\sigma}$ & $\boldsymbol{\lambda}$ & $\boldsymbol{S}$ & Closed Form & Integration \\
\hline 1 & $2 \%$ & $8 \%$ & $3 \%$ & $1 \%$ & $20 \%$ & $2 \%$ & 0.5 & 0.08498966 & 0.08498954 \\
1 & $2 \%$ & $8 \%$ & $3 \%$ & $1 \%$ & $20 \%$ & $2 \%$ & 1 & 0.02326991 & 0.02326994 \\
1 & $2 \%$ & $8 \%$ & $3 \%$ & $1 \%$ & $20 \%$ & $2 \%$ & 2 & 0.00363245 & 0.00363241 \\
1 & $6 \%$ & $8 \%$ & $3 \%$ & $1 \%$ & $20 \%$ & $2 \%$ & 1 & 0.04547443 & 0.04547454 \\
1 & $2 \%$ & $5 \%$ & $3 \%$ & $1 \%$ & $20 \%$ & $2 \%$ & 1 & 0.07619048 & 0.07618925 \\
1 & $2 \%$ & $8 \%$ & $1 \%$ & $1 \%$ & $20 \%$ & $2 \%$ & 1 & 0.01214452 & 0.01214454 \\
1 & $2 \%$ & $8 \%$ & $3 \%$ & $4 \%$ & $20 \%$ & $2 \%$ & 1 & 0.04300611 & 0.04300616 \\
1 & $2 \%$ & $8 \%$ & $3 \%$ & $1 \%$ & $40 \%$ & $2 \%$ & 1 & 0.07425119 & 0.07425130 \\
1 & $2 \%$ & $8 \%$ & $3 \%$ & $1 \%$ & $20 \%$ & $10 \%$ & 1 & 0.01096458 & 0.01096464 \\
\hline \hline
\end{tabular}

\section{Applicability of the Analytic Formulas}

Once the formulas in equations (14), (22) and (24) have been found, it is important to know under what circumstances they can be used in practical calculations. In the following sections, we address some of the primary issues associated with the use of these formulas.

\subsection{Applicability of the Mortality Laws}

One of the primary drawbacks of the derived formulas is the reliance on simplistic mortality laws in their derivation. We therefore tested the formulas against more realistic mortality represented by the 2000 Group Annuity Mortality table.

\subsubsection{Tests of the Constant Force of Mortality Formulas}

We will begin by comparing results of equation (24) with results from the 2000 Group Annuity Mortality table. The initial parameters for $r, p, q, \sigma$ and $\lambda$ used are those in the first row of Table 3 . The value of $S$ is allowed to vary from 0 to 2 in steps of 0.1 and the value of $\mu$ is age dependent and is set such that the value from equation (24) and the value found by integrating equation (1) for the realistic mortality are equal at $S=0$. The results at ages 40 and 65 are shown in Table 4 . The values at age 65 are in quite good agreement with a constant force approximation. However, the values at age 40 show significant deviations. In particular, although the values at $S=0$ have been set equal, the slopes have not been, and the slopes are matched much more closely at age 65 than at age 40. This suggests adjusting two parameters in order to match both the value and the slope at $S=0$. The most reasonable parameters to adjust are the mortality and lapse rates, which now become age dependent. 
TABLE 4.

Comparison of Constant Force Equation and 2000 Group Annuity Mortality at Age 40 and 65

\begin{tabular}{|c|c|c|c|c|c|c|c|c|c|c|}
\hline Strike & $\mu$ & Age & $r$ & $p$ & $q$ & $\sigma$ & $\lambda$ & $S$ & Constant Force & 2000GAM \\
\hline 1 & $0.86 \%$ & 40 & $8 \%$ & $3 \%$ & $1 \%$ & $20 \%$ & $2 \%$ & 0 & 0.10912893 & 0.10912893 \\
\hline 1 & $0.86 \%$ & 40 & $8 \%$ & $3 \%$ & $1 \%$ & $20 \%$ & $2 \%$ & 0.1 & 0.09046434 & 0.08411324 \\
\hline 1 & $0.86 \%$ & 40 & $8 \%$ & $3 \%$ & $1 \%$ & $20 \%$ & $2 \%$ & 0.2 & 0.07506622 & 0.06776989 \\
\hline 1 & $0.86 \%$ & 40 & $8 \%$ & $3 \%$ & $1 \%$ & $20 \%$ & $2 \%$ & 0.3 & 0.06188460 & 0.05548495 \\
\hline 1 & $0.86 \%$ & 40 & $8 \%$ & $3 \%$ & $1 \%$ & $20 \%$ & $2 \%$ & 0.4 & 0.05052989 & 0.04565157 \\
\hline 1 & $0.86 \%$ & 40 & $8 \%$ & $3 \%$ & $1 \%$ & $20 \%$ & $2 \%$ & 0.5 & 0.04077308 & 0.03747935 \\
\hline 1 & $0.86 \%$ & 40 & $8 \%$ & $3 \%$ & $1 \%$ & $20 \%$ & $2 \%$ & 0.6 & 0.03245779 & 0.03050981 \\
\hline 1 & $0.86 \%$ & 40 & $8 \%$ & $3 \%$ & $1 \%$ & $20 \%$ & $2 \%$ & 0.7 & 0.02546816 & 0.02444959 \\
\hline 1 & $0.86 \%$ & 40 & $8 \%$ & $3 \%$ & $1 \%$ & $20 \%$ & $2 \%$ & 0.8 & 0.01971373 & 0.01909827 \\
\hline 1 & $0.86 \%$ & 40 & $8 \%$ & $3 \%$ & $1 \%$ & $20 \%$ & $2 \%$ & 0.9 & 0.01512125 & 0.01431246 \\
\hline 1 & $0.86 \%$ & 40 & $8 \%$ & $3 \%$ & $1 \%$ & $20 \%$ & $2 \%$ & 1 & 0.01162979 & 0.00999499 \\
\hline 1 & $0.86 \%$ & 40 & $8 \%$ & $3 \%$ & $1 \%$ & $20 \%$ & $2 \%$ & 1.2 & 0.00731340 & 0.00740959 \\
\hline 1 & $0.86 \%$ & 40 & $8 \%$ & $3 \%$ & $1 \%$ & $20 \%$ & $2 \%$ & 1.4 & 0.00494076 & 0.00570200 \\
\hline 1 & $0.86 \%$ & 40 & $8 \%$ & $3 \%$ & $1 \%$ & $20 \%$ & $2 \%$ & 1.6 & 0.00351763 & 0.00451331 \\
\hline 1 & $0.86 \%$ & 40 & $8 \%$ & $3 \%$ & $1 \%$ & $20 \%$ & $2 \%$ & 1.8 & 0.00260681 & 0.00365348 \\
\hline 1 & $0.86 \%$ & 40 & $8 \%$ & $3 \%$ & $1 \%$ & $20 \%$ & $2 \%$ & 2 & 0.00199385 & 0.00301206 \\
\hline 1 & $4.72 \%$ & 65 & $8 \%$ & $3 \%$ & $1 \%$ & $20 \%$ & $2 \%$ & 0 & 0.40288709 & 0.40288709 \\
\hline 1 & $4.72 \%$ & 65 & $8 \%$ & $3 \%$ & $1 \%$ & $20 \%$ & $2 \%$ & 0.1 & 0.34438793 & 0.34147526 \\
\hline 1 & $4.72 \%$ & 65 & $8 \%$ & $3 \%$ & $1 \%$ & $20 \%$ & $2 \%$ & 0.2 & 0.29099806 & 0.28708288 \\
\hline 1 & $4.72 \%$ & 65 & $8 \%$ & $3 \%$ & $1 \%$ & $20 \%$ & $2 \%$ & 0.3 & 0.24260656 & 0.23967309 \\
\hline 1 & $4.72 \%$ & 65 & $8 \%$ & $3 \%$ & $1 \%$ & $20 \%$ & $2 \%$ & 0.4 & 0.19915553 & 0.19822801 \\
\hline 1 & $4.72 \%$ & 65 & $8 \%$ & $3 \%$ & $1 \%$ & $20 \%$ & $2 \%$ & 0.5 & 0.16060496 & 0.16179181 \\
\hline 1 & $4.72 \%$ & 65 & $8 \%$ & $3 \%$ & $1 \%$ & $20 \%$ & $2 \%$ & 0.6 & 0.12692423 & 0.12957252 \\
\hline 1 & $4.72 \%$ & 65 & $8 \%$ & $3 \%$ & $1 \%$ & $20 \%$ & $2 \%$ & 0.7 & 0.09808852 & 0.10092763 \\
\hline 1 & $4.72 \%$ & 65 & $8 \%$ & $3 \%$ & $1 \%$ & $20 \%$ & $2 \%$ & 0.8 & 0.07407701 & 0.07533536 \\
\hline 1 & $4.72 \%$ & 65 & $8 \%$ & $3 \%$ & $1 \%$ & $20 \%$ & $2 \%$ & 0.9 & 0.05487173 & 0.05236943 \\
\hline 1 & $4.72 \%$ & 65 & $8 \%$ & $3 \%$ & $1 \%$ & $20 \%$ & $2 \%$ & 1 & 0.04045691 & 0.03171702 \\
\hline 1 & $4.72 \%$ & 65 & $8 \%$ & $3 \%$ & $1 \%$ & $20 \%$ & $2 \%$ & 1.2 & 0.02353173 & 0.02116412 \\
\hline 1 & $4.72 \%$ & 65 & $8 \%$ & $3 \%$ & $1 \%$ & $20 \%$ & $2 \%$ & 1.4 & 0.01488257 & 0.01482926 \\
\hline 1 & $4.72 \%$ & 65 & $8 \%$ & $3 \%$ & $1 \%$ & $20 \%$ & $2 \%$ & 1.6 & 0.01000730 & 0.01078103 \\
\hline 1 & $4.72 \%$ & 65 & $8 \%$ & $3 \%$ & $1 \%$ & $20 \%$ & $2 \%$ & 1.8 & 0.00705154 & 0.00807261 \\
\hline 1 & $4.72 \%$ & 65 & $8 \%$ & $3 \%$ & $1 \%$ & $20 \%$ & $2 \%$ & 2 & 0.00515568 & 0.00619253 \\
\hline
\end{tabular}

The value of the GMDB option at $S=0$ can be found by substitution into equation (1). It turns out that

$$
f_{a}(0,0)=\int_{0}^{\infty} X e^{p w} e^{-r w} e^{-\lambda w} \mu_{x}(w)_{w} p_{x} d w=\frac{\mu}{\mu+r+\lambda-p}
$$

The slope $\Delta=\frac{\partial f_{a}}{\partial S}$ at $S=0$ can be found by differentiating the inside of the integral in equation (1) and then substituting $S=0$. So, 
TABLE 5

Comparison of Constant Force Equation and 2000 Group Annuity Mortality at Age 40 and 65 With Matched Slopes

\begin{tabular}{|c|c|c|c|c|c|c|c|c|c|c|c|}
\hline Strike & $\mu$ & Age & $r$ & $p$ & $q$ & $\sigma$ & $\lambda$ & $\operatorname{dj} \lambda$ & $S$ & Closed Form & 000GAM \\
\hline 1 & 0 & 40 & $8 \%$ & $3 \%$ & $1 \%$ & 2070 & $2 \%$ & & 0 & & 0.10912893 \\
\hline 1 & $0.65 \%$ & 40 & $8 \%$ & $3 \%$ & $1 \%$ & $20 \%$ & $2 \%$ & $0.33 \%$ & 0.1 & 0.08783152 & 08411324 \\
\hline 1 & $0.65 \%$ & 40 & $8 \%$ & $3 \%$ & $1 \%$ & $20 \%$ & $2 \%$ & $0.33 \%$ & 0.2 & 0.07190695 & 06776989 \\
\hline 1 & $0.65 \%$ & 40 & $8 \%$ & $3 \%$ & $1 \%$ & $20 \%$ & $2 \%$ & $0.33 \%$ & 0.3 & 0.05885151 & 0.05548495 \\
\hline 1 & $0.65 \%$ & 40 & $8 \%$ & $3 \%$ & $1 \%$ & $20 \%$ & $2 \%$ & & 0.4 & & 0.04565157 \\
\hline 1 & $0.65 \%$ & 40 & $8 \%$ & $3 \%$ & $1 \%$ & $20 \%$ & $2 \%$ & 0 & 0.5 & 64 & 7935 \\
\hline 1 & $0.65 \%$ & 40 & $8 \%$ & $3 \%$ & $1 \%$ & $20 \%$ & $2 \%$ & $0.33 \%$ & 0.6 & 32 & 981 \\
\hline 1 & $0.65 \%$ & 40 & $8 \%$ & $3 \%$ & $1 \%$ & $20 \%$ & $2 \%$ & $0.33 \%$ & 0.7 & 0.02459377 & 0.02444959 \\
\hline 1 & $0.65 \%$ & 40 & $8 \%$ & $3 \%$ & $1 \%$ & $20 \%$ & $2 \%$ & & 0.8 & 8 & 9827 \\
\hline 1 & $0.65 \%$ & 40 & $8 \%$ & $3 \%$ & $1 \%$ & $20 \%$ & $2 \%$ & $0.33 \%$ & 0.9 & 73 & 246 \\
\hline 1 & $0.65 \%$ & 40 & $8 \%$ & $3 \%$ & $1 \%$ & $20 \%$ & $2 \%$ & $0.33 \%$ & 1 & 0.0 & 499 \\
\hline 1 & $0.65 \%$ & 40 & $8 \%$ & $3 \%$ & $1 \%$ & $20 \%$ & $2 \%$ & $0.33 \%$ & 1.2 & & 0959 \\
\hline 1 & $0.65 \%$ & 40 & $8 \%$ & $3 \%$ & $1 \%$ & $20 \%$ & $2 \%$ & $0.33 \%$ & 1.4 & 23 & 0.00570200 \\
\hline 1 & $0.65 \%$ & 40 & $8 \%$ & $3 \%$ & $1 \%$ & $20 \%$ & $2 \%$ & 0.3 & 1.6 & 6 & 51331 \\
\hline 1 & $0.65 \%$ & 40 & $8 \%$ & $3 \%$ & $1 \%$ & $20 \%$ & $2 \%$ & & 1.8 & & 348 \\
\hline 1 & $0.65 \%$ & 40 & $8 \%$ & $3 \%$ & $1 \%$ & $20 \%$ & $2 \%$ & $0.33 \%$ & 2 & 91 & 0.003 \\
\hline 1 & $4.41 \%$ & 65 & $8 \%$ & $3 \%$ & $1 \%$ & $20 \%$ & $2 \%$ & 1. & 0 & 0.402 & 0.40288709 \\
\hline 1 & $4.41 \%$ & 65 & $8 \%$ & $3 \%$ & $1 \%$ & $20 \%$ & $2 \%$ & 1. & 0.1 & 0.3 & 7526 \\
\hline 1 & $4.41 \%$ & 65 & $8 \%$ & $3 \%$ & $1 \%$ & $20 \%$ & $2 \%$ & & 0.2 & & 0.28708288 \\
\hline 1 & $4.41 \%$ & 65 & $8 \%$ & $3 \%$ & $1 \%$ & $20 \%$ & $2 \%$ & $1.53 \%$ & 0.3 & 0.24 & 0.23967309 \\
\hline 1 & $4.41 \%$ & 65 & $8 \%$ & $3 \%$ & $1 \%$ & $20 \%$ & $2 \%$ & $1.53 \%$ & 0.4 & 0.19710314 & 0.19822801 \\
\hline 1 & $4.41 \%$ & 65 & $8 \%$ & $3 \%$ & $1 \%$ & $20 \%$ & $2 \%$ & $1.53 \%$ & 0.5 & 0.15894350 & 0.16179181 \\
\hline 1 & $4.41 \%$ & 65 & $8 \%$ & $3 \%$ & $1 \%$ & $20 \%$ & $2 \%$ & & 0.6 & & 0.12957252 \\
\hline 1 & $4.41 \%$ & 65 & $8 \%$ & $3 \%$ & $1 \%$ & $20 \%$ & $2 \%$ & $1.53 \%$ & 0.7 & 29 & 0.10092763 \\
\hline 1 & $4.41 \%$ & 65 & $8 \%$ & $3 \%$ & $1 \%$ & $20 \%$ & $2 \%$ & $1.53 \%$ & 0.8 & 0.07390323 & 0.07533536 \\
\hline 1 & $4.41 \%$ & 65 & $8 \%$ & $3 \%$ & $1 \%$ & $20 \%$ & $2 \%$ & $1.53 \%$ & 0.9 & 0.05509348 & 0.05236943 \\
\hline 1 & $4.41 \%$ & 65 & $8 \%$ & $3 \%$ & $1 \%$ & $20 \%$ & $2 \%$ & & 1 & 0.04094380 & 0.03171702 \\
\hline 1 & $4.41 \%$ & 65 & $8 \%$ & $3 \%$ & $1 \%$ & $20 \%$ & $2 \%$ & $1.53 \%$ & 1.2 & 0.02416882 & 0.02116412 \\
\hline 1 & $4.41 \%$ & 65 & $8 \%$ & $3 \%$ & $1 \%$ & $20 \%$ & $2 \%$ & $1.53 \%$ & 1.4 & 0.01547731 & 0.01482926 \\
\hline 1 & $4.41 \%$ & 65 & $8 \%$ & $3 \%$ & $1 \%$ & $20 \%$ & $2 \%$ & $1.53 \%$ & 1.6 & 0.01052026 & 0.01078103 \\
\hline 1 & $4.41 \%$ & 65 & $8 \%$ & $3 \%$ & $1 \%$ & $20 \%$ & $2 \%$ & $1.53 \%$ & 1.8 & 0.00748396 & 0.00807261 \\
\hline 1 & $4.41 \%$ & 65 & $8 \%$ & $3 \%$ & $1 \%$ & $20 \%$ & $2 \%$ & $1.53 \%$ & 2 & 0.00551868 & 0.00619253 \\
\hline
\end{tabular}

$$
\Delta(0,0)=\left.\frac{\partial f_{a}}{\partial S}\right|_{S=0}=-\int_{0}^{\infty} e^{-q w} e^{-\lambda w} \mu_{x}(w)_{w} p_{x} d w=-\frac{\mu}{\mu+q+\lambda}
$$

The two equations for $\mu$ and $\lambda$ solve for:

$$
\lambda=\frac{(r-p)[1+\Delta(0,0)] f_{a}(0,0)+q\left[1-f_{a}(0,0)\right] \Delta(0,0)}{-\Delta(0,0)-f_{a}(0,0)}
$$


TABLE 6.

Comparison of DeMoivre's LaW Equation and 2000 Group Annuity Mortality at Age 40 and 65 WITH MATCHED SLOPES

\begin{tabular}{|c|c|c|c|c|c|c|c|c|c|c|c|}
\hline Strike & Age & $r$ & $p$ & $q$ & $\sigma$ & $\lambda$ & $\operatorname{Adj} \lambda$ & $T-t$ & $S$ & DeMoivre's Law & 2000GAM \\
\hline 1 & 40 & $8 \%$ & $3 \%$ & $1 \%$ & $20 \%$ & $2 \%$ & $0.82 \%$ & 157.33 & 0 & 0.10912893 & 0.109129 \\
\hline 1 & 40 & $8 \%$ & $3 \%$ & $1 \%$ & $20 \%$ & $2 \%$ & $0.82 \%$ & 157.33 & 0.1 & 08753263 & 13 \\
\hline 1 & 40 & $8 \%$ & $3 \%$ & $1 \%$ & $20 \%$ & $2 \%$ & $0.82 \%$ & 157.33 & 0.2 & .07156195 & 0.067770 \\
\hline 1 & 40 & $8 \%$ & $3 \%$ & $1 \%$ & $20 \%$ & $2 \%$ & $0.82 \%$ & 157.33 & 0.3 & 0.05852753 & 0.055485 \\
\hline 1 & 40 & $8 \%$ & $3 \%$ & $1 \%$ & $20 \%$ & $2 \%$ & $0.82 \%$ & 157.33 & 0.4 & 0.04764948 & 0.045652 \\
\hline 1 & 40 & $8 \%$ & $3 \%$ & $1 \%$ & $20 \%$ & $2 \%$ & $0.82 \%$ & 157.33 & 0.5 & 0.03851424 & 0.037479 \\
\hline 1 & 40 & $8 \%$ & $3 \%$ & $1 \%$ & $20 \%$ & $2 \%$ & $0.82 \%$ & 157.33 & 0.6 & 0.03085899 & 0.030510 \\
\hline 1 & 40 & $8 \%$ & $3 \%$ & $1 \%$ & $20 \%$ & $2 \%$ & $0.82 \%$ & 157.33 & 0.7 & 0.024 & 0.024450 \\
\hline 1 & 40 & $8 \%$ & $3 \%$ & $1 \%$ & $20 \%$ & $2 \%$ & $0.82 \%$ & 157.33 & 0.8 & 0.01929858 & 0.019098 \\
\hline 1 & 40 & $8 \%$ & $3 \%$ & $1 \%$ & $20 \%$ & $2 \%$ & $0.82 \%$ & 157.33 & 0.9 & 12 & 0.014312 \\
\hline 1 & 40 & $8 \%$ & $3 \%$ & $1 \%$ & $20 \%$ & $2 \%$ & $0.82 \%$ & 157.33 & 1 & 12 & 0.009995 \\
\hline 1 & 40 & $8 \%$ & $3 \%$ & $1 \%$ & $20 \%$ & $2 \%$ & $0.82 \%$ & 157.33 & 1.2 & 809 & 0.007410 \\
\hline 1 & 40 & $8 \%$ & $3 \%$ & $1 \%$ & $20 \%$ & $2 \%$ & $0.82 \%$ & 157.33 & 1.4 & 0.00555902 & 0.005702 \\
\hline 1 & 40 & $8 \%$ & $3 \%$ & $1 \%$ & $20 \%$ & $2 \%$ & $0.82 \%$ & 157.33 & 1.6 & .00410086 & 0.004513 \\
\hline 1 & 40 & $8 \%$ & $3 \%$ & $1 \%$ & $20 \%$ & $2 \%$ & $0.82 \%$ & 157.33 & 1.8 & .00313569 & 0.003653 \\
\hline 1 & 40 & $8 \%$ & $3 \%$ & $1 \%$ & $20 \%$ & $2 \%$ & $0.82 \%$ & 157.33 & 2 & 0.00 & 0.003012 \\
\hline 1 & 65 & $8 \%$ & $3 \%$ & $1 \%$ & $20 \%$ & $2 \%$ & $2.24 \%$ & 30.54 & 0 & 0.40288709 & 0.402887 \\
\hline 1 & 65 & $8 \%$ & $3 \%$ & $1 \%$ & $20 \%$ & $2 \%$ & $2.24 \%$ & 30.54 & 0.1 & 0.34083990 & 0.341475 \\
\hline 1 & 65 & $8 \%$ & $3 \%$ & $1 \%$ & $20 \%$ & $2 \%$ & $2.24 \%$ & 30.54 & 0.2 & .28468545 & 0.287083 \\
\hline 1 & 65 & $8 \%$ & $3 \%$ & $1 \%$ & $20 \%$ & $2 \%$ & $2.24 \%$ & 30.54 & 0.3 & 0.23 & 0.239673 \\
\hline 1 & 65 & $8 \%$ & $3 \%$ & $1 \%$ & $20 \%$ & $2 \%$ & $2.24 \%$ & 30.54 & 0.4 & 0.19201047 & 0.198228 \\
\hline 1 & 65 & $8 \%$ & $3 \%$ & $1 \%$ & $20 \%$ & $2 \%$ & $2.24 \%$ & 30.54 & 0.5 & 0.15467650 & 0.161792 \\
\hline 1 & 65 & $8 \%$ & $3 \%$ & $1 \%$ & $20 \%$ & $2 \%$ & $2.24 \%$ & 30.54 & 0.6 & 0.12276945 & 0.129573 \\
\hline 1 & 65 & $8 \%$ & $3 \%$ & $1 \%$ & $20 \%$ & $2 \%$ & $2.24 \%$ & 30.54 & 0.7 & 0.09592391 & 0.100928 \\
\hline 1 & 65 & $8 \%$ & $3 \%$ & $1 \%$ & $20 \%$ & $2 \%$ & $2.24 \%$ & 30.54 & 0.8 & 0.07382662 & 0.075335 \\
\hline 1 & 65 & $8 \%$ & $3 \%$ & $1 \%$ & $20 \%$ & $2 \%$ & $2.24 \%$ & 30.54 & 0.9 & 0.05620810 & 0.052369 \\
\hline 1 & 65 & $8 \%$ & $3 \%$ & $1 \%$ & $20 \%$ & $2 \%$ & $2.24 \%$ & 30.54 & 1 & 0.04283475 & 0.031717 \\
\hline 1 & 65 & $8 \%$ & $3 \%$ & $1 \%$ & $20 \%$ & $2 \%$ & $2.24 \%$ & 30.54 & 1.2 & 0.02636432 & 0.021164 \\
\hline 1 & 65 & $8 \%$ & $3 \%$ & $1 \%$ & $20 \%$ & $2 \%$ & $2.24 \%$ & 30.54 & 1.4 & 0.01738788 & 0.014829 \\
\hline 1 & 65 & $8 \%$ & $3 \%$ & $1 \%$ & $20 \%$ & $2 \%$ & $2.24 \%$ & 30.54 & 1.6 & 0.01206547 & 0.010781 \\
\hline 1 & 65 & $8 \%$ & $3 \%$ & $1 \%$ & $20 \%$ & $2 \%$ & $2.24 \%$ & 30.54 & 1.8 & 0.00870532 & 0.008073 \\
\hline 1 & 65 & $8 \%$ & $3 \%$ & $1 \%$ & $20 \%$ & $2 \%$ & $2.24 \%$ & 30.54 & 2 & 0.00647835 & 0.006193 \\
\hline
\end{tabular}

and

$$
\mu=(q+\lambda)\left[\frac{-\Delta(0,0)}{1+\Delta(0,0)}\right]
$$

$\mu$ and $\lambda$ now both depend on the issue age of the policy. The results with matched slopes are given in Table 5. The agreement is quite good. The most substantial deviations occur around $S=1$, which suggests that the formula is of more use in valuation than in pricing. 
We now compare the results of equation (14) with the 2000 GAM table. We expect greater agreement in this case because DeMoivre's Law is a better approximation of human mortality in the sense that mortality rates rise with age and there is a maximum age as well. Again, the initial parameters for $r, p$, $q, \sigma$ and $\lambda$ used are those in the first row of Table 3. The value of $S$ is allowed to vary from 0 to 2 in steps of 0.1 . Finally, we vary both $T-t$ and $\lambda$ to match both the values and slopes at $S=0$ and the results are presented in Table 6 .

\subsection{Stochastic Interest Rates}

Equations (1) and (3) are both based on the idea that yield curves are flat and do not change over time. If the yield curve is not flat, but future interest rates are completely determined by today's forward rates, equation (1) is changed only marginally to:

$$
\begin{aligned}
& f_{a}(S, t)= \\
& \int_{0}^{\infty}\left\{X e^{p t} e^{p w} e^{-{ }_{w} r_{t} w} N\left[-d_{2}(S, w)\right]-S e^{-q w} N\left[-d_{1}(S, w)\right]\right\} \mu(t+w)_{w} p_{x+t}^{(\tau)} d w
\end{aligned}
$$

where ${ }_{w} r_{t}$ represents the forward rate of interest for cash flows invested at time $t$ and maturing at time $t+w$. We will now check equation (24) against a numerical evaluation of equation (32) for a non-level yield curve. The initial parameters for $p, q, \sigma$ and $\lambda$ used are those in the first row of Table 3, and the starting yield curve is ${ }_{t} r_{0}=.08-.04 e^{-t / 10}$. We will set the rate $r=7.14 \%$ in equation (24) to be the one that sets the two equations equal at $S=0$. The agreement is quite good, with a maximum deviation of 0.0034 . Checking equation (14) against equation (32) under similar conditions yields similarly good agreement.

Values of put options when interest rates are stochastic were first considered by Merton (1973). Rabinovitch (1989) derives option prices for the specific case where interest rates follow a constant volatility mean reverting process. This is unlikely to have a large effect, as Kim and Kunitomo (1999) claim that "the trend of interest rate plays a crucial role in determining the stock option values. The effects of the volatility of interest rate process as well as the correlation between the stock price and interest rate are of secondary importance". We have already examined the effect of interest rate trends and shown it to be relatively unimportant. Stochastic hazard rates have a similarly small effect if the mortality yield curve implied in market prices of annuities is used in the formulas.

It may seem at this point that fitting to numerical evaluations do not result in any gain from using the closed form solutions. The advantage comes when a model needs to rapidly compute a large number of option prices. In this case, the parameters in the closed form can be precomputed and used repeatedly, which could result in a considerable time savings. 


\subsection{Sensitivity of Results to Parameter Values}

We now show the sensitivity of the equations to their parameters for a reasonable set of starting values. We used the constant force of mortality formula in Equation (24) and took numerical derivatives with respect to six parameters. The results are shown in figure 1 . The graph shows the change in the value of the option for a $1 \%$ change in the parameter values for various market levels. The value of the option increases with an increase in most parameter values, but decreases with an increase in lapse rates or the risk-free rate. Therefore, we plot the absolute values of these derivatives to facilitate comparison.

When the GMDB is in-the-money, the option value is most sensitive to changes in mortality and lapse rates. In addition, the lapse rate is the most difficult parameter to estimate. When the GMDB is out-of-the-money, the volatility and the risk-free rate become more important than the lapse and mortality rate. However, these economic parameters can be estimated much more easily than the lapse rate. The risk-free rate is available from the current yield curve, and the volatility can be inferred from traded option values. It appears that good estimates of the lapse rate, while difficult, are necessary for accurate valuation of GMDB riders. Next, we use Table 4 and Figure 1 to compare the errors introduced by using a simplified mortality law to the errors introduced by uncertainty in parameter values. The errors in Table 4 are of the order of 0.0001 to 0.001 . This is the same general range of $1-10 \%$ errors in parameter values. That is, using a lapse rate of $10 \%$ in valuation when the experience lapse rate is $11 \%$ (a $10 \%$ change) is at least as important as the error introduced by using a simplified mortality law.

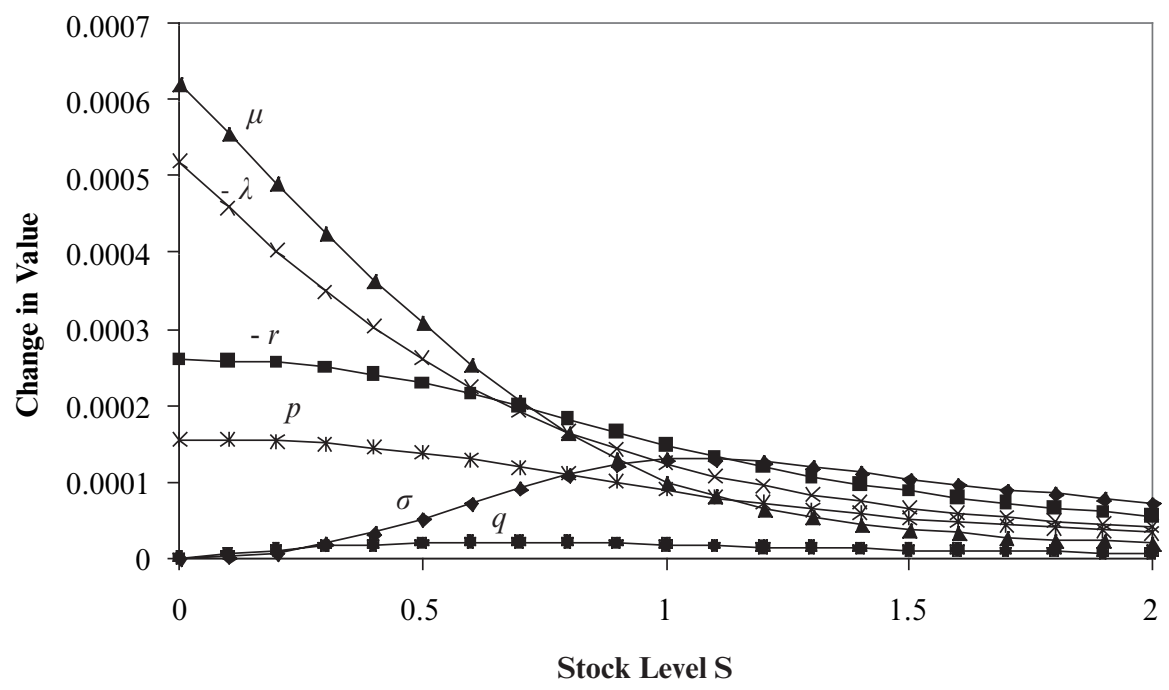

FIgure 1: Sensitivity of the Constant Force Equation to its Parameters. The Base Values are $\mu=0.0086, r=5 \%, p=3 \%, q=1 \%, \sigma=20 \%$ and $\lambda=10 \%$. 


\section{Conclusions}

In this paper, we have solved the differential equation that must be satisfied for a rollup GMDB attached to a variable annuity contract for two well-known mortality laws. Solutions have been found previously only in the case of constant force of mortality and rollup interest rate $p=0 \%$. This paper extends the results to positive $p$, as well as providing a solution for term insurance and endowment insurance under constant force of mortality. In addition, solutions for the GMDB value under DeMoivre's law mortality are derived. The answers compare well with past work, as well as answers obtained from commonly used numerical integration methods.

\section{Appendix A. Derivation of Equations (4)-(6)}

Equation (3) can be rewritten as:

$$
\begin{gathered}
\frac{\partial f_{a}}{\partial t}-\left[r+\mu_{x}(t)+\lambda\right] f_{a}+(r-q) S \frac{\partial f_{a}}{\partial S}+\frac{1}{2} \sigma^{2} S^{2} \frac{\partial^{2} f_{a}}{\partial S^{2}}= \\
-\left[\mu_{x}(t)\right]\left[X e^{p t}-S\right] H\left(X e^{p t}-S\right)
\end{gathered}
$$

where $H(x)$ is the Heaviside step function, whose derivative is the Dirac delta function $\delta(x)$. Now, I will assume $f_{a}(S, t)$ is of the form:

$$
f_{a}(S, t)=\left\lfloor X e^{p t} A(t)-S B(t)\right\rfloor H\left(X e^{p t}-S\right)+C(S, t)
$$

Substituting Equation (A2) into Equation (A1) gives:

$$
\begin{aligned}
& \left.\left\{\mid X e^{p t} A^{\prime}(t)-(r+\mu(t)+\lambda-p) X e^{p t} A(t)\right]+\left[-S B^{\prime}(t)+(q+\mu(t)+\lambda) S B(t)\right]\right\} H\left(X e^{p t}-S\right) \\
& +\left\{-X e^{p t} S A(t)(r-q)+\left(r-q+\sigma^{2}\right) S^{2} B(t)+p X^{2} e^{2 p t} A(t)-p X e^{p t} S B(t)\right\} \delta\left(X e^{p t}-S\right) \\
& +\left\{\frac{1}{2} \sigma^{2} X e^{p t} S^{2} A(t)-\frac{1}{2} \sigma^{2} S^{3} B(t)\right\} \delta^{\prime}\left(X e^{p t}-S\right) \\
& +\frac{\partial C}{\partial t}-\left[r+\mu_{x}(t)+\lambda\right] C+(r-q) S \frac{\partial C}{\partial S}+\frac{1}{2} \sigma^{2} S^{2} \frac{\partial^{2} C}{\partial S^{2}}=\left[-\mu_{x}(t) X e^{p t}+\mu_{x}(t) S\right] H\left(X e^{p t}-S\right)
\end{aligned}
$$

The heaviside functions can be canceled and the right hand side of equation (A3) can be set equal to 0 if

$$
A^{\prime}(t)-(r+\mu(t)+\lambda-p) A(t)=-\mu(t)
$$


and

$$
B^{\prime}(t)-(q+\mu(t)+\lambda) B(t)=-\mu(t)
$$

Bowers et al page 125 (1997) states that

$$
\frac{d}{d x} \bar{A}_{x}-(\delta+\mu(x)) \bar{A}_{x}=-\mu(x)
$$

where $\delta$ is the force of interest and $\bar{A}_{x}$ is the net single premium for a whole life policy on a person age $x$. This implies that $A(t)$ and $B(t)$ are $\bar{A}_{x+t}$ at force of interest $r+\lambda-p$ and $q+\lambda$ respectively.

$C$ must now obey the following equation:

$$
\begin{aligned}
& \frac{\partial C}{\partial t}-\left[r+\mu_{x}(t)+\lambda\right] C+(r-q) S \frac{\partial C}{\partial S}+\frac{1}{2} \sigma^{2} S^{2} \frac{\partial^{2} C}{\partial S^{2}} \\
& =\left\{(r-q) X e^{p t} S A(t)-\left(r-q+\sigma^{2}\right) S^{2} B(t)-p X^{2} e^{2 p t} A(t)+p X e^{p t} S B(t)\right\} \delta\left(X e^{p t}-S\right) \\
& +\left\{\frac{1}{2} \sigma^{2} S^{3} B(t)-\frac{1}{2} \sigma^{2} X e^{p t} S^{2} A(t)\right\} \delta^{\prime}\left(X e^{p t}-S\right)
\end{aligned}
$$

The left hand side is similar to Equation (3) but the right hand side now has sources related to the delta function and its derivatives. We now make Equation (A7) dimensionless. The derivation follows similar lines to that found in Wilmott et al. (1995). We pick dimensionless variables $y=\ln \left(\frac{S}{X}\right)$ and $\tau=\frac{\sigma^{2}(T-T)}{2} . T$ is currently an arbitrary parameter as, unlike the case for the vanilla European options, there is no expiration date to the GMDB option. Later, we will see that $T$ represents the age at which the GMDB must be exercised. For a mortality function such as de Moivre's law with a built in maximum age, $T$ represents the time remaining until that age is reached. For a mortality function without a maximum age, $T$ can be viewed as the time until the GMDB is no longer a death benefit, but an endowment benefit. If there is no endowment age, we will let $T \rightarrow \infty$ as the final step. We will assume $C(S, t)=$ $X e^{a y} f(\tau) w(y, \tau)$ :

We chose $\alpha$ to be $\frac{1}{2}-\frac{(r-q)}{\sigma^{2}}$, define $\beta$ to be $\frac{2 p}{\sigma^{2}}$, and assume that $f(\tau)$ satisfies:

$$
f^{\prime}(\tau)+\left[\frac{2\left(r+\mu_{x}(\tau)+\lambda\right)}{\sigma^{2}}+\alpha^{2}\right] f(\tau)=0
$$

Being careful with the delta functions (see Barton (1989)), this leads to the following equation for $w(y, \tau)$ : 


$$
\begin{aligned}
\frac{\partial w}{\partial \tau}-\frac{\partial^{2} w}{\partial y^{2}} & =\left\{(3-2 \alpha) e^{(2-\alpha) y} e^{-p T} e^{\beta \tau} \frac{B(\tau)}{f(\tau)}-(1-2 \alpha) e^{(1-\alpha) y} \frac{A(\tau)}{f(\tau)}\right. \\
& \left.+\beta e^{-a y} e^{p T} e^{-\beta \tau} \frac{A(\tau)}{f(\tau)}-\beta e^{(1-\alpha) y} \frac{B(\tau)}{f(\tau)}\right\} \delta(y-[p T-\beta \tau]) \\
& +\left\{e^{(2-\alpha) y} e^{-p T} e^{\beta \tau} \frac{B(\tau)}{f(\tau)}-e^{(1-\alpha) y} \frac{A(\tau)}{f(\tau)}\right\} \delta^{\prime}(y-[p T-\beta \tau])
\end{aligned}
$$

The ordinary differential equation for $f(\tau)$ is solved by:

$$
f(\tau)=f\left(\tau_{0}\right) e^{-\kappa\left(\tau-\tau_{0}\right)} e^{-\frac{2}{\sigma^{2}} \int_{\tau_{0}}^{\tau} \mu(s) d s}
$$

where $\kappa=\frac{2(r+\lambda)}{\sigma^{2}}+\alpha^{2}, \tau_{0}$ and $f\left(\tau_{0}\right)$ are arbitrary constants, and $\mu(s)$ is the functional form of $\mu(\tau)$ not $\mu(t)$.

Equation (A9) is a diffusion equation with sources. The solution is:

$$
\begin{aligned}
w(y, \tau) & =\int_{0}^{\tau} \int_{-\infty}^{\infty} \frac{e^{\beta s} \frac{B(s)}{f(s)}}{\sqrt{4 \pi(\tau-s)}}(3-2 \alpha) e^{-p T} e^{(2-\alpha) \xi} e^{-(y-\xi)^{2} / 4(\tau-s)} \delta(\xi-[p T-\beta s]) d \xi d s \\
& +\int_{0}^{\tau} \int_{-\infty}^{\infty} \frac{\frac{A(s)}{f(s)}}{\sqrt{4 \pi(\tau-s)}}(2 \alpha-1) e^{(1-\alpha) \xi} e^{-(y-\xi)^{2} / 4(\tau-s)} \delta(\xi-[p T-\beta s]) d \xi d s \\
& +\int_{0}^{\tau} \int_{-\infty}^{\infty} \frac{e^{-\beta s \frac{A(s)}{f(s)}}}{\sqrt{4 \pi(\tau-s)}} \beta e^{p T} e^{-\alpha \xi} e^{-(y-\xi)^{2} / 4(\tau-s)} \delta(\xi-[p T-\beta s]) d \xi d s \\
& -\int_{0}^{\tau} \int_{-\infty}^{\infty} \frac{\frac{B(s)}{f(s)}}{\sqrt{4 \pi(\tau-s)}} \beta e^{(1-\alpha) \xi} e^{-(y-\xi)^{2} / 4(\tau-s)} \delta(\xi-[p T-\beta s]) d \xi d s \\
& +\int_{0}^{\tau} \int_{-\infty}^{\infty} \frac{e^{\beta s} \frac{B(s)}{f(s)}}{\sqrt{4 \pi(\tau-s)}} e^{-p T} e^{(2-\alpha) \xi} e^{-(y-\xi)^{2} / 4(\tau-s)} \delta^{\prime}(\xi-[p T-\beta s]) d \xi d s \\
& -\int_{0}^{\tau} \int_{-\infty}^{\infty} \frac{\frac{A(s)}{f(s)}}{\sqrt{4 \pi(\tau-s)}} e^{(1-\alpha) \xi} e^{-(y-\xi)^{2} / 4(\tau-s)} \delta^{\prime}(\xi-[p T-\beta s]) d \xi d s
\end{aligned}
$$

assuming $w(y, 0)=0$. This is the same condition as

$$
f_{a}(S, T)=\left\lfloor X e^{p T} A(T)-S B(T)\right\rfloor H\left(X e^{p T}-S\right)
$$

implying that $T$ is either the age at which mortality is $100 \%$, or is an endowment age that can be allowed to increase to infinity. 
Integrating over the delta functions gives:

$$
\begin{aligned}
w(y, \tau) & =\frac{\alpha+\beta}{\sqrt{4 \pi}} e^{(1-\alpha) p T} \int_{0}^{\tau} e^{(\alpha-1) \beta s} \frac{A(s)}{f(s)} \frac{e^{-(y-[p T-\beta s])^{2} / 4(\tau-s)}}{\sqrt{\tau-s}} d s \\
& -\frac{\alpha+\beta-1}{\sqrt{4 \pi}} e^{(1-\alpha) p T} \int_{0}^{\tau} e^{(\alpha-1) \beta s} \frac{B(s)}{f(s)} \frac{e^{-(y-[p T-\beta s])^{2} / 4(\tau-s)}}{\sqrt{\tau-s}} d s \\
& +\frac{e^{(1-\alpha) p T}}{4 \sqrt{\pi}} \int_{0}^{\tau} e^{(\alpha-1) \beta s} \frac{A(s)}{f(s)}(y-[p T-\beta s]) \frac{e^{-(y-[p T-\beta s])^{2} / 4(\tau-s)}}{(\tau-s)^{3 / 2}} d s \\
& -\frac{e^{(1-\alpha) p T}}{4 \sqrt{\pi}} \int_{0}^{\tau} e^{(\alpha-1) \beta s} \frac{B(s)}{f(s)}(y-[p T-\beta s]) \frac{e^{-(y-[p T-\beta s])^{2} / 4(\tau-s)}}{(\tau-s)^{3 / 2}} d s s
\end{aligned}
$$

\section{REFERENCES}

Barton, G. (1989) Elements of Green's Functions and Propagation, New York: Oxford University Press.

Bowers, N.L., Gerber, H.U., Hickman, J.C., Jones, D.A. and Nesbitt, C.J. (1997) Actuarial Mathematics, Schaumburg, Illinois: The Society of Actuaries.

Hardy, M. (2003) Investment Guarantees, Hoboken, N.J.: John Wiley and Sons.

Kim, Y.J. and Kunitomo, N. (1999) Pricing Options under Stochastic Interest Rates: A New Approach, Asia-Pacific Financial Markets 6, 49-70.

Merton, R.C. (1973) Theory of Rational Option Pricing, Bell Journal of Economics and Management Science 4(1), 141-183.

Milevsky, M.A. and Posner, S.E. (2001) The Titanic Option: Valuation of the Guaranteed Minimum Death Benefit in Variable Annuities and Mutual Funds, Journal of Risk and Insurance 68(1), 93-128.

Milevsky, M.A. and Salisbury, T.S. (2001) The Real Option to Lapse and the Valuation of DeathProtected Investments, Conference Proceedings of the 11th Annual International AFIR Colloquium, Sep 2001, p. 537.

Rabinovitch, R. (1989) Pricing Stock and Bond Options when the Default Free Rate is Stochastic, Journal of Financial and Quantitative Analysis 24(4), 447-457.

Ulm, E.R. (2006) The Effect of the Real Option to Transfer on the Value of Guaranteed Minimum Death Benefits, Journal of Risk and Insurance 73(1), 43-69.

Wilmott, P., Howison, S. and DeWynne, J. (1995) The Mathematics of Financial Derivatives, Cambridge, England: Cambridge.

\section{ERIC R. ULM}

Department of Risk Management and Insurance

J. Mack Robinson College of Business

Georgia State University, Atlanta, GA.

Tel.: 404-413-7485.

E-mail: inseuu@langate.gsu.edu. 